\title{
Probiotics alleviate inflammatory bowel disease in mice by regulating intestinal microorganisms-bile acid-NLRP3 inflammasome pathway
}

\author{
Zongshi Liu'1,2 and Hong Wang ${ }^{1,2}$ \\ 1Department of Internal Medicine, The First Affiliated Hospital of Jinan University, Guangzhou, Guangdong, China; 2Department of Internal \\ Medicine, Guangzhou First People's Hospital, School of Medicine, South China University of Technology, Guangzhou, Guangdong, China
}

The objective of the present study was to elucidate the mechanism of intestinal microorganisms-bile acid-NLRP3 inflammasome regulation in mice with inflammatory bowel disease treated with probiotics. The abnormal activation of NLRP3 inflammasome is the main pathogenic factor that leads to the development of chronic colitis in IL-10-/- mice. In this study, we divided the IL-10-/- and wild-type mice on a C57BL/6 background into 3 groups: control group (wt mice, $\mathrm{n}=10$ ), IBD group (IL-10-/- mice, $\mathrm{n}=10$ ), and probiotic group (IL-10-/- mice treated with probiotics, $n=10$ ). The analyses included mRNA levels of cytokines and protein expression of NLRP3 inflammasome and NOD2, as well as colorimetric determination of Wnt, Notch and BMP activity in colon tissue and fresh colon mass. The fresh colon mass was increased in the IBD mice when compared with the control and the probiotic groups $(P<0.05)$. The histological score of the proximal colon in the IBD group was higher than in two other groups $(P<0.05)$. The probiotic group showed lower levels of IFN- $\gamma$, IL-17F, IL-1 $\alpha$ and IL-25 mRNA compared to the IBD group $(P<0.05)$. The main components of NLRP3 inflammasome (NLRP3, ASC, caspase-1 and IL-1 $\beta$ ) and NOD2 were increased in IBD group compared to the control, and decreased after probiotic treatment $(P<0.05)$. FXR, TGR5, vitamin $D$, and CAR were all increased in IBD group compared to the control and probiotic groups $(P<0.05)$. In conclusion, probiotics modulated the intestinal microbialbile acid-NLRP3 inflammation in IBD mice.

Keywords: probiotics; inflammatory bowel disease; intestinal microorganisms; bile acid; NLRP3 inflammasomes axis

Received: 18 January, 2021; revised: 10 February, 2021; accepted: 20 July, 2021; available on-line: 14 October, 2021

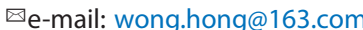

Abbreviations: CAR, constitutive androstane receptor; $C D, C r o h n ' s$ disease; IBD, Inflammatory bowel disease; NLRs, Nucleotide-binding and oligomerization domain-1ike receptors; UC, ulcerative colitis; PXR, pregnane $X$ receptor; VDR, vitamin D receptor

\section{INTRODUCTION}

Inflammatory bowel disease (IBD), including ulcerative colitis (UC) and Crohn's disease (CD), is caused by gastrointestinal mucosal immune dysfunction. Interleukin-10 deficient (IL-10-/-) mouse model is one of the most widely used animal models of IBD (Sokol et al., 2018). People with IL-10R mutation can spontaneously develop into Crohn's disease patients at a very young age ( $<1$ year), which further emphasizes the importance of IL-10 signal transduction and supports the use of IL$10^{-/-}$colitis mouse model to understand the pathogen- esis of IBD, especially the correlation with CD (Qazi et al., 2017).

Nucleotide-binding and oligomerization domain-1ike receptors (NLRs) are intracellular innate immune receptors, forming a family of at least 22 members in human and 33 members in mouse. These receptors are considered to play an important role in the interaction between host and microorganisms. The interaction between host and environment, as well as immune regulation, has attracted great attention in the field of intestinal mucosal immunity and inflammation. NLRP3 is one of the most typical members of NLRP (Cannito et al., 2017). NLRP3 can react with a variety of pathogens, immunogens, or endogenous danger signals. Research shows that there is a mechanism of IBD related to genetic variation and mutation of NLRP3 gene.

The intestinal flora is composed of many kinds of bacteria and plays an important role in the health of the host. Probiotics genera in human body are mainly Lactobacillus, Bifidobacterium, Bacillus, and some butyric acid bacteria (Hai, 2015). The signal molecules produced by intestinal flora include, among others, short chain fatty acids, lipopolysaccharides, flagellin, and peptidoglycans. They can interact with specific host receptors and affect a number of host processes, including energy metabolism, and immune function (Celiberto et al., 2018). Kawase et al. demonstrated that the fermentation products of Lactobacillus and Streptococcus could significantly reduce vasoconstrictive pressure, decrease total cholesterol level in blood and increase high density lipoprotein level (Kawase et al., 2000). Bifidobacterium and its surface molecules can induce tumor apoptosis and inhibit tumor growth through the expression of genes related to tumor cell apoptosis; NAD oxidoreductase and SOD produced by Bifidobacterium can help enhance the body's anti-cancer ability (Ouwehand et al., 2002). The gut microflora also plays a key role in the chemical modification of bile acids, which may ultimately influence their local and systemic signaling. The main role of bile acids is to digest lipids in the digestive tract, but they also have a signaling function similar to that of endocrine hormones. Changes in the balance of microbial species in the gut (occurring for example in malnutrition) can change the metabolic potential of the community and lead to changes in the characteristics of bile acids, ultimately causing a host response. Many recent studies have analyzed the changes in bile acid signaling in the development of gastrointestinal and parenteral diseases and related these findings to microbial abnormalities.

Probiotics are live microorganisms that provide health benefits when consumed. Probiotics usually have 
a long history of safe use, and many of them have been approved as GRAS (generally recognized as safe) by FDA. The ultimate standard of a probiotic is that it is scientifically proven to be effective in promoting health or preventing and treating specific diseases (Huang et al., 2019). Probiotic preparation VSL\#3 is a drug commonly used in experimental and clinical research in recent years. VSL\#3 is a lyophilized bacterial mixture containing4 Lactobacillus strains, 3 Bifidobacterium strains and Streptococcus thermophilus, in the amount of $9 \times 10^{11}$ CFU bacteria in each portion. Tursi et al. summarized the recent studies on the use of VSL\#3 in combination with low doses of mesalazine in the treatment of IBD and proposed that this method had positive impact on the treatment of mild to moderate ulcerative colitis (Tursi et al., 2007). On the other hand, a controlled study of 30 CD patients treated with Synbiotic 2000 (a mixture of 4 probiotic species and 4 prebiotics) or placebo after surgery showed that two years later there was no difference in clinical symptoms, laboratory tests and endoscopy results between the two groups (Chermesh et al., 2007).

In this experiment we selected a probiotic mixture containing Lactobacillus rhamnosus dm905 (Quality Inspection Center of Chinese Medicine, China) and Lactococcus lactis JCM 5805 (Zhongke Quality Inspection Strain Preservation Center, China) to explore its effect on the intestinal microbial bile acid-NLRP3 inflammation pathway in mice with IBD.

\section{MATERIALS AND METHODS}

\section{Animal experiments}

C57BL/6 IL-10-/- mice and C57BL/ 6 wild type mice were kept at controlled temperature $\left(25^{\circ} \mathrm{C}\right)$ and photoperiod with SPF (12:12-h light-dark cycle). The mice were divided into three experimental groups of ten according to their genotype and feeding regime. The control group consisted of C57BL/6 wild type mice supplied with ordinary drinking water; the IBD group consisted of C57BL/6 IL-10-/- mice supplied with ordinary drinking water and the probiotic group consisted of C57BL/6 IL-10-/- mice that were given probiotics at $100 \mathrm{mg} / \mathrm{kg} /$ day during the course of experiment. The probiotic used was a mixed microbial flora (the number of viable bacteria $\leq 2 \times 10^{10} \mathrm{CFU} / \mathrm{g}$ ) containing Lactobacillus rhamnosus dm905 (quality inspection center of Chinese medicine, China) and Lactococcus lactis JCM 5805 (Zhongke Quality Inspection Strain Preservation Center, China). All the mice were sacrificed at experimental day 8 and their colons were immediately extracted for further analyses.

\section{Ethics}

This study protocol has been approved by Animal Care and Use Committee of Guangzhou First People's Hospital (Approval number is DWGZ-2019-048).

\section{Histological evaluation of colitis}

Proximal and distal colon sections were stained with hematoxylin and eosin to examine colon inflammation and damage. Histological scores were assigned by the pathologists unaware of the treatment conditions. Each colon segment (proximal or distal) was scored on a scale of 0 to 8 (0 meaning no inflammation and 8 meaning severe damagewith a combined score of 0 to 8 per animal.

\section{RNA isolation and real-time PCR}

RNA was extracted from the colon mucosa of mice using Trizol (Invitrogen, Carlsbad, CA), and reverse transcribed using High-Capacity cDNA Reverse Transcription Kit (Applied Biosystems, Carlsbad, CA). PCR amplification was performed using $12.5 \mu \mathrm{L}$ Dream'Taq ${ }^{\mathrm{TM}}$ Green PCR Master Mix (Fermentas-Glen Burnie-MD, USA). Primer sequences for RT-qPCR are listed in Supplementary Table 1 (at https://ojs.ptbioch. edu.pl/index.php/abp/).

\section{Western blotting assay}

The colon protein extracts were separated on SDSpolyacrylamide gel and transferred onto $0.2 \mu \mathrm{m}$ polydiene-difluoro-ether membrane (Millipore, Bedford, MA, USA). The membrane was blocked with 3, BSA (MP Biomedicals, Irvine, CA, USA) in TBST for $\times h$ and incubated with IL-1 $\beta$, caspase-1, or $\beta$-actin antibodies (Sigma-Aldrich, St. USA) in $4^{\circ} \mathrm{C}$ overnight, in or in RT. The protein blots were developed with Clarity ${ }^{\mathrm{TM}}$ Western System (BIO-RAD, CA, USA) and photographed using the BIO-RAD ChemiDoc ${ }^{\mathrm{TM}}$ XRS System.

\section{Wnt, Notch and BMP activity determination}

The colon tissue lysate was centrifuged and the protein in the supernatant was quantified. Forty $\mu \mathrm{L}$ of caspase- 1 reaction buffer and $50 \mu \mathrm{L}$ of each sample $(100 \mu \mathrm{g}$ protein) were added to 96 well plate, and $10 \mu \mathrm{L}$ of substrate was added. After incubation at $37^{\circ} \mathrm{C}$ for 2 hours, the activity of Wnt, Notch and BMP was measured by spectrophotometry at $405 \mathrm{~nm}$.

\section{Statistical Analysis}

The statistical software package SPSS 14.0 (SPSS Inc., Chicago, IL, USA) was used to analyze the data. Students' $t$-test was used to compare two groups, and the results were expressed as mean \pm 688 standard error. The data were also evaluated by one-way ANOVA with Tukey's post hoc multiple comparison test; $P<0.05$ was considered statistically significant.

\section{RESULTS}

\section{Morphological features of chronic colitis in IL-10-/- mice}

Colitis in mice is characterized by enlargement and thickening of the colon wall, diarrhea, or poor formation of fecal particles, and rectal prolapse. We found that the fresh colon weight could be used as an accurate indicator of IBD in IL-10-/- mice. Fresh colon weight in the

Table 1. Colon weight statistics,$- \mathrm{x} \pm \mathrm{s}$

\begin{tabular}{ll}
\hline Group & Fresh weight $(\mathrm{g})$ \\
\hline Control & $0.25 \pm 0.06$ \\
\hline IBD & $0.68 \pm 0.11$ \\
\hline Probiotics & $0.37 \pm 0.04$ \\
\hline$F$ & 12.356 \\
\hline$P$ value & 0.014 \\
\hline
\end{tabular}



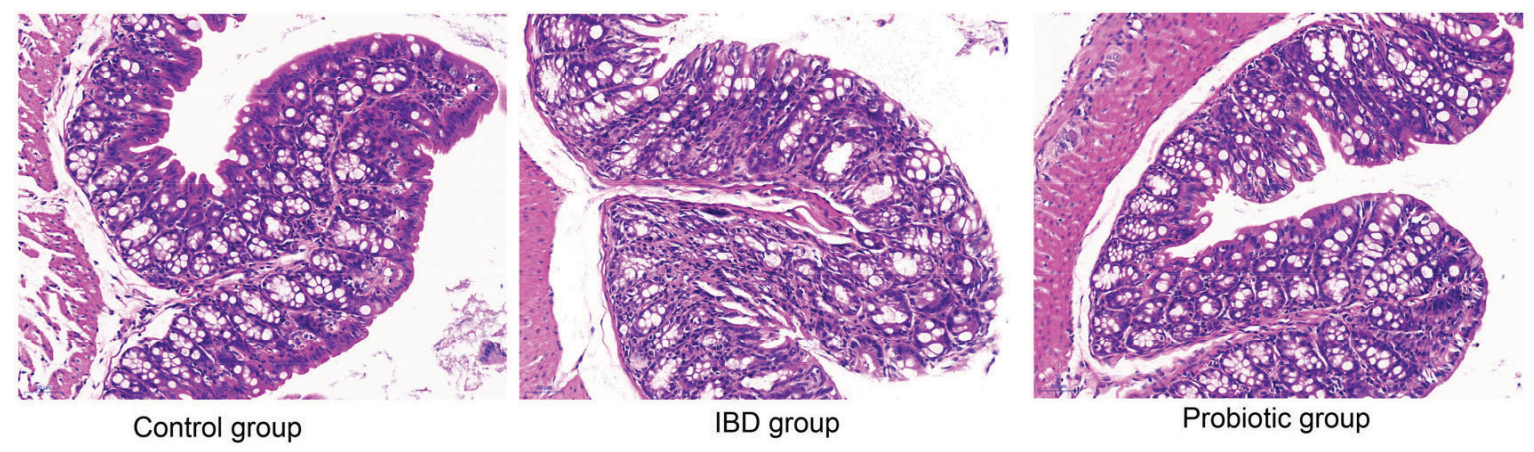

Figure 1. Histological examination of the colon using hematoxylin and eosin staining

IBD group was higher than in the control group, while that in the probiotics group it was lower than that in the IBD group $(P<0.05)$. Data are shown in Table 1.

\section{Molecular characteristics of IL-10-/- mice with chronic colitis}

Histological colon analysis showed clear microscopic lesions with goblet cell atrophy, crypt loss, and excessive regenerative proliferation or leukocyte infiltration in mice with colitis. Probiotic therapy relieved colon disease in mice (Fig. 1). The histological score of the proximal colon in the IBD group was higher than in the control group, and in the probiotic group it was lower than in the IBD group $(P<0.05)$. Data are summarized in Table 2 .

\section{Expression of cytokines mRNA}

Probiotic-fed mice's histology significantly differed from their IL-10-/- counterparts, so we measured the mRNA expression of a variety of cytokines in the control (?), IBD and probiotic groups. Two electrophoretic

Table 2. Histological analysis of the proximal colon,$- x \pm s$

\begin{tabular}{ll}
\hline Group & Score \\
\hline Control & $1.00 \pm 0.32$ \\
\hline IBD & $7.29 \pm 0.16$ \\
\hline Probiotics & $3.17 \pm 0.27$ \\
\hline$F$ & 13.964 \\
\hline$P$ value & $<0.001$ \\
\hline
\end{tabular}

Table 3. Expression level of cytokine mRNA -, $x \pm s$

\begin{tabular}{|c|c|c|c|c|c|}
\hline Cytokine & Control & $\mathrm{IBD}$ & Probiotics & $\mathrm{F}$ & $P$ value \\
\hline IL-1a & $0.24 \pm 0.06$ & $5.88 \pm 0.48$ & $0.37 \pm 0.08$ & 13.524 & $<0.001$ \\
\hline IL-25 & $0.45 \pm 0.22$ & $8.76 \pm 0.34$ & $0.53 \pm 0.09$ & 11.546 & $<0.001$ \\
\hline IL-17F & $0.68 \pm 0.14$ & $7.96 \pm 0.33$ & $0.66 \pm 0.37$ & 12.879 & $<0.001$ \\
\hline IFN- $\gamma$ & $0.35 \pm 0.04$ & $9.88 \pm 0.78$ & $0.37 \pm 0.11$ & 10.337 & $<0.001$ \\
\hline TNF- $a$ & $0.27 \pm 0.16$ & $8.81 \pm 0.35$ & $0.37 \pm 0.08$ & 9.264 & $<0.001$ \\
\hline IL-18 & $0.15 \pm 0.03$ & $8.78 \pm 0.24$ & $0.31 \pm 0.17$ & 11.356 & $<0.001$ \\
\hline IL-22R1 & $9.43 \pm 0.82$ & $9.47 \pm 0.79$ & $9.46 \pm 0.85$ & 13.542 & 0.379 \\
\hline
\end{tabular}

bands in each one were repeated in two mice. They represent at least four mice in each group. In IL-10-/- mice, we detected increased levels of pro-inflammatory cytokines mRNA that included IL-18, TNF- $\alpha$, IFN- $\gamma$ and IL-17F. Compared with the control group, their blood lipids also increased $(P<0.05)$. The probiotic group showed lower IFN- $\gamma$, IL-17F, IL-1 $\alpha$ and IL-25 mRNA levels than the IBD group $(P<0.05)$. The expression of IL-22 receptor-1 (il-22r1), was used as a control, stayed constant between the three groups $(P>0.05)$. Data are shown in Table 3.

\section{Probiotics increase the constitutive expression of NLRP3 in $\mathrm{IL}-10^{-/-}$mice}

cDNA gel electrophoresis and Western blot of the total lysates revealed the main expression components of NLRP3 inflammasomes, including NLRP3, ASC, caspase 1 , and IL-1 beta in the IL10-/- mice. Since NOD2 is a member of the NLRP family and one of the most characteristic IBD related genes, we examined its protein expression alongside NLRP3, ASC, caspase 1, and IL-1 in the control, IBD and probiotic groups. Compared with the control group, both NOD2 and all the major components of NLRP3 inflammatory bodies were increased in IL-10-/- mice (IBD group) and decreased in the group treated with probiotics $(P<0.05)$ (Fig. 2 and Table 4).

\section{Probiotics increase the expression of nuclear receptors in IL-10-/- mice}

Bile acid activated nuclear receptors such as pregnane $\mathrm{X}$ receptor (PXR), G-protein-coupled bile acid receptor (TGR5), vitamin $\mathrm{D}$ receptor (VDR) and constitutive androstane receptor (CAR) play a key role in the regulation of lipid, glucose, and energy metabolism, 


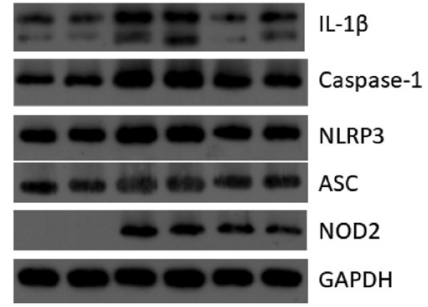

Figure 2. Probiotics increase constitutive expression of NLRP3 inflammasomes in IL-10-/- mice

inflammation and drug metabolism and detoxification. Expression of nuclear receptors in the control IBD and probiotic groups was compared. PXR, TGR5, VDR and CAR were increased in IL-10-/- IBD group mice and decreased in the probiotic group $(P<0.05)$ (Table 5$)$.

\section{Probiotics affect the relative number of gut microbes}

The relative numbers of gut microbes were compared between the mice from the control, IBD and probiotic groups. Mice from the IBD group (IL-10-/-) had elevated relative numbers of Mycobacterium, sulfur-reducing bacteria, Lactobacillus and Bacillus, and decreased relative numbers of Enterobacteriaceae compared to the control group. In the group of $\mathrm{IL}-10^{-/-}$mice that were receiving probiotics, the relative numbers of Mycobacterium, sulfur-reducing bacteria, Lactobacillus and Bacillus were decreased, and the relative number of Enterobacteriaceae was increased compared to the IBD group $(P<0.05)$. Data are shown in Table 6.

\section{Probiotics increase expression of Wnt, Notch, b-actin, and BMP in IL-10-/- mice}

We studied the expression of major components of the Wnt, Notch, and BMP pathways in mice from the control, IBD and probiotic groups. Wnt, Notch, $\beta$-catenin and BMP were decreased in IBD group compared to the control, but increased in probiotic group compared to IBD group $(P<0.05)$. Data are shown in Table 7.

\section{DISCUSSION}

There is a strong evidence that intestinal flora is involved in the pathogenesis of IBD. In the experiment presented here, the fresh colon weight there was significantly higher in the IBD group than in the probiotic group. The colon of IBD mice showed significant wall thickening This result supported the significance of probiotics in the treatment of IBD.

Histological score of 0 points is assigned to a colon with a normal tissue structure, 1 point to a slight submucosal or lamina propria separation, and a score greater than or equal to 2 can be described as necrotizing colitis. Therefore the lower the score, the healthier the intestine. In this experiment, the histological scores of the colon extracted from the probiotic-fed IL-10-/- mice were sig-

Table 4. Expression level of NLRP3 inflammasomes,$- x \pm s$

\begin{tabular}{llllll}
\hline Cytokine & Control & IBD & Probiotics & $F$ & $P$ value \\
\hline IL-1 $\beta$ & $0.57 \pm 0.23$ & $4.88 \pm 0.46$ & $1.24 \pm 0.23$ & 12.251 & $<0.001$ \\
\hline Caspase 1 & $0.46 \pm 0.12$ & $5.88 \pm 0.38$ & $1.31 \pm 0.28$ & 11.329 & $<0.001$ \\
\hline NLRP3 & $0.62 \pm 0.11$ & $8.74 \pm 0.47$ & $1.58 \pm 0.27$ & 9.324 & $<0.001$ \\
\hdashline ASC & $1.56 \pm 0.34$ & $8.36 \pm 0.42$ & $2.71 \pm 0.73$ & 12.338 & $<0.001$ \\
\hline NOD2 & $0.27 \pm 0.07$ & $1.43 \pm 0.16$ & $0.38 \pm 0.14$ & 13.145 & $<0.001$ \\
\hline
\end{tabular}

Table 5. Expression level of nuclear receptor,$- x \pm s$

\begin{tabular}{llllll}
\hline Nuclear receptor & Control & IBD & Probiotics & $F$ & $P$ value \\
\hline FXR & $3.42 \pm 0.31$ & $0.73 \pm 0.17$ & $2.89 \pm 0.24$ & 12.371 & 0.013 \\
\hline TGR5 & $4.48 \pm 0.28$ & $0.68 \pm 0.14$ & $3.95 \pm 0.32$ & 11.375 & 0.014 \\
\hline vit-D & $5.26 \pm 0.19$ & $1.23 \pm 0.17$ & $4.88 \pm 0.42$ & 14.372 & 0.011 \\
\hline CAR & $5.22 \pm 0.31$ & $0.97 \pm 0.15$ & $4.95 \pm 0.16$ & 10.876 & $<0.001$ \\
\hline
\end{tabular}

Table 6. Relative quantity of Gut microbes,$- x \pm s$

\begin{tabular}{llllll}
\hline Gut microbes & Control & IBD & Probiotics & F & $P$ value \\
\hline Mycobacterium & $100.37 \pm 10.24$ & $128.45 \pm 11.07$ & $107.24 \pm 9.58$ & 12.435 & 0.021 \\
\hline Sulfur reducing bacteria & $100.42 \pm 3.56$ & $124.53 \pm 12.48$ & $105.32 \pm 8.59$ & 11.483 & 0.033 \\
\hline Lactobacillus & $100.85 \pm 8.57$ & $122.46 \pm 13.54$ & $106.42 \pm 7.83$ & 13.157 & 0.015 \\
\hdashline Bacillus & $100.42 \pm 7.53$ & $132.28 \pm 15.17$ & $108.78 \pm 9.89$ & 15.768 & 0.014 \\
\hline Enterobacteriaceae & $100.43 \pm 6.26$ & $87.27 \pm 7.54$ & $97.43 \pm 6.74$ & 13.82 & 0.015 \\
\hline
\end{tabular}


Table 7. Expression level of Wnt, Notch, $\beta$-catenin, and BMP -, $\mathbf{x} \pm$

\begin{tabular}{llllll}
\hline Parameters & Control & IBD & Probiotics & $F$ & $P$ value \\
\hline Wnt & $3.33 \pm 0.21$ & $0.67 \pm 0.13$ & $2.74 \pm 0.32$ & 12.318 & 0.015 \\
\hdashline Notch & $4.67 \pm 0.38$ & $1.22 \pm 0.12$ & $4.03 \pm 0.35$ & 11.326 & 0.013 \\
\hline$\beta$-catenin & $7.24 \pm 0.17$ & $2.55 \pm 0.27$ & $6.78 \pm 0.75$ & 13.627 & 0.014 \\
\hdashline BMP & $5.16 \pm 0.31$ & $0.82 \pm 0.15$ & $4.53 \pm 0.56$ & 11.426 & 0.007 \\
\hline
\end{tabular}

nificantly lower than for the mice form the IBD group, indicating that the intestinal tissue of mice fed with probiotics was healthier (Nadler et al., 2000).

Probiotics can achieve their therapeutic effects in IBD in a number of ways. They influence the composition of the intestinal flora and change the metabolic characteristics of the microbiome (Hayase \& Hashimoto, 2017). By increasing the production of short chain fatty acids, they can lower the $\mathrm{pH}$ of the colon's environment, thereby inhibiting the growth of potential pathogenic microorganisms. Many probiotic strains can produce antibacterial substances, such as hydrogen peroxide, hydrogen sulfide, lactic acid and specific bacteriocins, and replace harmful microorganisms by competing for binding sites on the lumen mucosa interface (Zhang et al., 2018).

Each probiotic strain may have unique immune regulation characteristics, so probiotics can indirectly or directly regulate intestinal immune response. In short, the effects of probiotics on the immune system can be divided into two categories: one with immune stimulating properties, the other with anti-inflammatory properties. The down regulation of inflammatory immune response by probiotics including those with clinical efficacy in IBD treatment, was shown by many studies (Nothaft et al., 2017). Several probiotics work by enhancing regulatory $\mathrm{T}$ cells (Tregs) response. Tregs are antigen-specific $\mathrm{T}$ cells, which can prevent autoimmunity and maintain tolerance to harmless antigens (including intestinal symbiotic flora). They can control the excessive activation of the NF- $x \mathrm{~B}$ pathway, reduce the production of proinflammatory cytokines (such as TNF- $\alpha, I N F_{-} \gamma$ and IL-8), and induce the production and secretion of antiinflammatory cytokines (such as IL-10 and TGF- $\beta$ ) (Liu et al., 2017). In our study we got similar results. The expression of the inflammatory factors IFN- $\gamma$, IL-17F, IL$1 \alpha$ and IL-25 was significantly reduced in the probiotic group as compared to the ID mice.

In the past two decades, a number of interventional clinical studies have been published to compare the efficacy of probiotic therapy versus placebo or standard therapy versus medication. With different study designs and different probiotic strains and doses, only a few studies are similar so that the results can be compared consistently, so it is difficult to come to common conclusions.

The changes in the spectrum of bile acids and the microbial population in IBD patients in remission or flare state have been analyzed. Compared to the healthy controls, IBD remission in patients is characterized by a reduction in a number of bacteria from the Firmicutes phylum (especially Clostridium coccoides, C. leptum and Faecalibacterium prausnitzii) in the gut (Perera et al., 2017). In our study, the probiotic treatment resulted in a decrease of the relative number of mycobacteria, sulfur-reducing bacteria, lactobacilli, and Bacillus, while the relative number of Enterobacter increased $(P<0.05)$.
In the past decade, the composition of intestinal flora in IBD patients has been studied extensively. A large number of studies have found that the fecal microflora of IBD patients has different composition than that of the healthy people, and some differences between the microbial populations in $\mathrm{CD}$ and $\mathrm{UC}$ were also detected (Gonçalves et al., 2017). In addition, differences were observed between the active and inactive stages of the disease, and between the inflamed and non-inflamed areas of the intestine. There are certain facts to remember when studying the intestinal flora in IBD. First of all, only $30 \%$ of the total bacterial population can be identified with the conventional bacteriology technology, but the detection rate can be greatly improved by using the molecular technology, although a large number of bacteria remain not detected (Compton et al., 2017). Secondly, many strains found in the IBD patients' intestines do not belong to the main phylogenetic groups occurring in healthy people.

In addition, the mucosal and fecal flora should be analyzed separately.. The composition of these two domains is unique, and it seems to be important in IBD. In enteritis patients, especially CD patients, the concentration of mucosal bacteria was higher than in healthy controls. Fecal microflora differs from that associated with the mucosa; the latter may be more related to intestinal immune regulation (Zhang \& Jiang, 2017). A reduction in microbial diversity in IBD has been previously reported. This decrease is due to a large loss of bacterial species, including Eubacterium and Lactobacillus. The decrease in the number of mucosa-associated bifidobacteria and the increase in the number of E. coli and Clostridium in IBD patients support the hypothesis that an imbalance between potentially beneficial and pathogenic bacteria may lead to the development of this disease (Gao et al., 2018). The experimental results support this hypothesis. Macrogenomics was used to demonstrate that the complexity of Bacteroides, especially Clostridium, in CD patients was reduced compared to healthy controls. However, other studies have shown a correlation between the increase in the number of mucosae-associated bacteria and the increase in the number of bacteria from the Enterobacteriaceae family, including the invasive E. coli.

Data show that NLRP3 plays a key role in the pathogenesis of IBD in humans. Compared to the control group, NLRP3 inflammatory bodies were activated in vitro in $\mathrm{CD}$ patients, while in UC patients they were activated in the late stage of the disease. This finding was combined with an in vivo increase in the concentration of IL-1 $\beta$ in peripheral blood mononuclear cells of CD patients as compared to the UC patients and healthy controls. A recent study showed that NLRP3 components were up-regulated in CD and UC patients, based on the increased mRNA expression of NLRP3, IL-1 $\beta$, ASC and caspase- 1 in colon biopsy (Cattaneo et al., 2017). This result is related to the exacerbation of the disease. The 
inhibition of NLRP3 in CD patients leads to the inhibition of pro-inflammatory cytokines and chemokines, emphasizing the pathogenic role of abnormal activation of NLRP3 in the disease. In addition, IL-1 $\beta$ and IL-18 cytokines were increased in plasma and colon mucosa of IBD patients. The increase of IL-1 $\beta$ secretion in colon and macrophages of IBD patients promotes chronic intestinal inflammation and is related to the severity of the disease.

The clinical research on NLRP3 inflammasome's activity is limited. Probiotics are NLRP3 inhibitors that act by interfering with the secretion of IL-1 $\beta$ and the activation of caspase- 1 mediated by inflammatory corpuscles. They have been proved to be potential and safe drugs for the treatment of UC. Controversial data on NLRP3 activity in IBD reveal the complex and possibly diverse roles of NLRP3 inflammatory bodies in IBD. As high expression levels of the NLRP3 components have been observed in both in IBD mice and patients, NLRP3 activation appears to be a major feature of inflammation. The activation of NLRP3 inflammasomes is a key step in the inflammatory process, which leads to tissue damage and the development of IBD clinical manifestations. The pathogenicity of NLRP3 may be due to the increase or abnormality of the complex's activity. The activation of NLRP3 inflammasomes and the end point of this process (IL-1 $\beta$, IL-18, fever) seem to be promising treatment options and need further study. In our study, the main components of NLRP3 inflammasome and NOD2 were significantly decreased in the probiotic group compared to the IBD group. This result is similar to that of Schmitz and others (2015), indicating that probiotics can inhibit the expression of chronic metabolic inflammasomes (Liang et al., 2019, Schmitz et al., 2015).

Most of the genes found to be associated with an increased risk of IBD encode proteins that maintain the mucosal barrier and/or regulate the host immune system. The discovery of NOD2/CARD15 gene product's involvement in IBD is a major breakthrough in understanding the relationship between genetic susceptibility and IBD development. The gene encodes a protein, which belongs to a pattern recognition receptor family and is responsible for the recognition of microorganisms, as well as the induction and control of anti-microbial genes' expression. Genetic defects found in IBD and $C D$ patients may make them particularly susceptible to infection by intracellular bacteria such as Mycobacterium avium, Listeria monocytogenes and invasive E. coli. Mutations in Toll like receptor and card4/Nod1 receptor genes may also be associated with the increased susceptibility to IBD.

In conclusion, probiotics regulate the expression of Wnt, notch, $\beta$-catenin and BMP by lowering the levels of mRNA of proinflammatory cytokines (IFN- $\gamma$, IL-17F, IL- $1 \alpha$ and IL-25) and the main components of NLRP3 inflammasomes (NLRP3, ASC, caspase-1 and IL-1 $\beta$ ), as well as the level of NOD2. Our data provide preclinical evidence and molecular basis for the development of new IBD therapies targeting NLRP3 inflammatory ribosomes and their downstream signaling molecules.

\section{Acknowledgement}

None.

\section{Funding}

None.

\section{Conflict of interest}

The authors declare no conflict of interest.

\section{REFERENCES}

Cannito S, Morello E, Bocca C, Foglia B, Benetti E, Novo E, Chiazza F, Rogazzo M, Fantozzi R, Povero D, Sutti S, Bugianesi E, Feldstein A E, Albano E, Collino M, Parola M (2017) Microvesicles released from fat-laden cells promote activation of hepatocellular NLRP3 inflammasome: A pro-inflammatory link between lipotoxicity and non-alcoholic steatohepatitis. PLoS One 12: e0172575. https://doi.org/10.1371/journal.pone.0172575

Cattaneo A, Cattane N, Galluzzi S, Provasi S, Lopizzo N, Festari C, Ferrari C, Guerra U P, Paghera B, Muscio C, Bianchetti A, Volta G D, Turla M, Cotelli M S, Gennuso M, Prelle A, Zanetti O, Lussignoli G, Mirabile D, Bellandi D, Gentile S, Belotti G, Villani D, Harach T, Bolmont T, Padovani A, Boccardi M, Frisoni G B (2017) Association of brain amyloidosis with pro-inflammatory gut bacterial taxa and peripheral inflammation markers in cognitively impaired elderly. Neurobiol Aging 49: 60-68. https://doi.org/10.1016/j.neurobiolaging.2016.08.019

Celiberto LS, Graef FA, Healey GR, Bosman ES, Jacobson K, Sly LM, Vallance BA (2018) Inflammatory bowel disease and immunonutrition: novel therapeutic approaches through modulation of diet and the gut microbiome. Immunology 155: 36-52. https://doi. org/10.1111/imm.12939

Chermesh I, Tamir A, Reshef R, Chowers Y, Suissa A, Katz D, Gelber M, Halpern Z, Bengmark S, Eliakim R (2007) Failure of Synbiotic 2000 to prevent postoperative recurrence of Crohn's disease. Dig Dis Sci 52: 385-359. https://doi.org/10.1007/s10620-006-9549-7

Compton SR, Booth CJ, Macy JD (2017) Lack of effect of murine astrovirus infection on dextran sulfate-induced colitis in NLRP3deficient Mice. Comp Med 67: 400-406.

Gao F, Xiang HC, Li HP, Jia M, Pan XL, Pan HL, Li M (2018) Electroacupuncture inhibits NLRP3 inflammasome activation through CB2 receptors in inflammatory pain. Brain Behav Immun 67: 91-100. https://doi.org/10.1016/j.bbi.2017.08.004

Gonçalves AC, Ferreira LS, Manente FA, De Faria C, Polesi MC, De Andrade CR, Zamboni DS, Carlos IZ (2017) The NLRP3 inflammasome contributes to host protection during Sporothrix schenckii infection. 151: 154-166. https://doi.org/10.1111/imm.12719

Hai N V (2015) The use of probiotics in aquaculture. I Appl Microbiol 119: 917-935. https://doi.org/10.1111/jam.12886

Hayase E, Hashimoto D (2017) R-Spondin1 expands Paneth cells and prevents dysbiosis induced by graft-versus-host disease. J Exp Med 214: 3507-3518. https://doi.org/10.1084/jem.20170418

Huang W, Gan W, Huang A, Fu Y, Shang Y, Chen Y, Tian Z, Zhang Y, Fang G (2019) Efficacy of electroacupuncture combined with probiotics for depression and chronic diarrhea in patients and effect on serum inflammatory cytokines, NE and BDNF. Exp Ther Med 18: 3470-3474. https://doi.org/10.3892/etm.2019.7977

Kawase M, Hashimoto H, Hosoda M, Morita H, Hosono A (2000) Effect of administration of fermented milk containing whey protein concentrate to rats and healthy men on serum lipids and blood pressure. J Dairy Sci 83: 255-263. https://doi.org/10.3168/jds. S0022-0302 (00)74872-7

Liang Y, Liang S, Zhang Y, Deng Y, He Y, Chen Y, Liu C, Lin C, Yang Q (2019) Oral administration of compound probiotics ameliorates HFD-induced gut microbe dysbiosis and chronic metabolic inflammation via the $G$ protein-coupled receptor 43 in non-alcoholic fatty liver disease rats. Probiotics Antimicrob Proteins 11: 175-185. https://doi.org/10.1007/s12602-017-9378-3

Liu L, Dong Y, Ye M, Jin S, Yang J, Joosse M E, Sun Y, Zhang J, Lazarev M, Brant SR, Safar B, Marohn M, Mezey E, Li X (2017) The pathogenic role of NLRP3 inflammasome activation in inflammatory bowel diseases of both mice and humans. J Crohns Colitis 11: 737-750. https://doi.org/10.1093/ecco-jcc/jjw219

Nadler EP, Dickinson E, Knisely A, Zhang XR, Boyle P, Beer-Stolz D, Watkins SC, Ford HR (2000) Expression of inducible nitric oxide synthase and interleukin-12 in experimental necrotizing enterocolitis. J Surg Res 92: 71-77. https://doi.org/10.1006/jsre.2000.5877

Nothaft H, Perez-Muñoz M E, Gouveia G J \& Duar R M (2017) Coadministration of the campylobacter jejuni N-glycan-based vaccine with probiotics improves vaccine performance in broiler chickens. Appl Environ Microbiol 83. https://doi.org/10.1128/aem.0152317

Ouwehand AC, Salminen S, Isolauri E (2002) Probiotics: an overview of beneficial effects. Antonie V an Leeunenboek 82: 279-289

Perera AP, Kunde D, Eri R (2017) NLRP3 Inhibitors as potential therapeutic agents for treatment of inflammatory bowel disease. Curr Pharm Des 23: 2321-2327. https://doi.org/10.2174/1381612823666 170201162414

Qazi T, Amaratunga T, Barnes EL, Fischer M, Kassam Z, Allegretti JR (2017) The risk of inflammatory bowel disease flares after fe- 
cal microbiota transplantation: Systematic review and meta-analysis. Gut Microbes 8: 574-588. https://doi.org/10.1080/19490976.2017.13 53848

Schmitz S, Werling D, Allenspach K (2015) Effects of ex-vivo and in-vivo treatment with probiotics on the inflammasome in dogs with chronic enteropathy. PLoS One, 10, e0120779. https://doi. org/10.1371/journal.pone.0120779

Sokol H, Jegou S, Mcquitty C, Straub M, Leducq V, Landman C, Kirchgesner J, Le Gall G, Bourrier A, Nion-Larmurier I, Cosnes J, Seksik P, Richard ML, Beaugerie L (2018) Specificities of the intestinal microbiota in patients with inflammatory bowel disease and Clostridium difficile infection. Gut Microbes 9: 55-60. https://doi.org /10.1080/19490976.2017.1361092

Tursi A, Brandimarte G, Giorgetti G M, Elisei W, Aiello F (2007) Balsalazide and/or high-potency probiotic mixture (VSL\#3) in maintaining remission after attack of acute, uncomplicated diverticulitis of the colon. Int J Colorectal Dis 22: 1103-1108. https://doi. org/10.1007/s00384-007-0299-6

Zhang L, Qin Q, Liu M, Zhang X, He F, Wang G (2018) Akkermansia muciniphila can reduce the damage of gluco/lipotoxicity, oxidative stress and inflammation, and normalize intestine microbiota in streptozotocin-induced diabetic rats. Pathog Dis 76. https://doi. org $/ 10.1093 /$ femspd/fty028

Zhang Q, Jiang X (2017) MCL Plays an anti-inflammatory role in mycobacterium tuberculosis-induced immune response by inhibiting NF- $x \mathrm{~B}$ and NLRP3 inflammasome activation. 2017: 2432904. https://doi.org/10.1155/2017/2432904 